\title{
Survival rates of breast cancer and predictive factors: a hospital-based study from western Amazon area in Brazil
}

\author{
Sobrevida do câncer de mama e fatores preditivos: \\ um estudo de base hospitalar do oeste amazônico, Brasil
}

Ruth Helena Pimenta Fujimoto ${ }^{1}$

Rosalina Jorge Koifman ${ }^{2}$

Ilce Ferreira da Silva ${ }^{3}$

${ }^{1}$ Programa de PósGraduação em Saúde Pública, Universidade Federal do Acre. Rodovia BR 364 Km 04, Distrito Industrial. 69920-900 Rio Branco AC Brasil. helena_fujimoto@ hotmail.com

${ }^{2}$ Escola Nacional de Saúde Pública Sérgio Arouca, Fiocruz. Rio de Janeiro RJ Brasil.

${ }^{3}$ Instituto Fernandes Figueira, Fiocruz. Rio de Janeiro RJ Brasil.

\begin{abstract}
Breast cancer survival in Latin America countries is below Central European countries. Hospital-based breast cancer survival studies in western Amazon, Brazil, are lacking. This article aims to estimate hospital-based breast cancer survival in Rio Branco, Acre, and predictor factors. Hospital-based cohort study of all women diagnosed with breast cancer (2007-2012) was proceeded. Information were obtained from medical reports, and follow-up was until 2013. One-, 2- and 5- years breast cancer specific-survival were estimated by Kaplan-Meier method. Crude and adjusted Harzards Ratios (HR) were estimated by proportional Cox regression model. One-, 2-, and 5-year overall breast cancer survival were $95.5 \%, 83.7 \%$, and $87.3 \%$ respectively. Surgery combined to radiotherapy significantly affected 1-, 2-, and 5-year survival (99\%, 94\%, and $90.6 \%$, respectively) as compared to other treatments $(77 \%, 57.1 \%$, and $37.5 \%$, respectively). Comparing to surgery combined to radiotherapy treatment, surgery alone increased the risk of death, independently of age and stage $(H R=$ 7.23;95\%CI:2.29-22.83). In Rio Branco, Acre, 5 -year breast cancer survival is similar to more developed areas in Brazil. Surgery combined to radiotherapy was independently associated to a lower risk of death as compared to surgery alone and other treatment.
\end{abstract}

Key words Breast cancer survival, Cancer treatment, Amazon
Resumo Na América Latina, a sobrevida de 5 anos do câncer de mama está abaixo dos países da Europa central. Não há estudos de sobrevida de câncer de mama de base hospitalar no oeste da Amazônia brasileira. O objetivo deste artigo é estimar a sobrevida hospitalar de câncer de mama e fatores associados em Rio Branco, Acre. Estudo de coorte de base hospitalar com todos os casos de câncer de mama diagnosticados em Rio Branco (2007-2012). As informações foram obtidas de prontuários. As sobrevidas específicas para câncer de mama foram estimadas para 1, 2, e 5 anos pelo método de Kaplan Meier. As hazards ratios (HR) brutas e ajustadas foram estimadas pela regressão proporcional de Cox. As sobrevidas globais em 1, 2 e 5 anos foram respectivamente 95,5\%, $83,7 \%$ e $87,3 \%$. A cirurgia combinada à radioterapia afetou significativamente a sobrevida em 1 , 2 e 5 anos (99\%, 94\% e 90,6\%, respectivamente) quando comparadas a outros tratamentos (77\%, $57,1 \%$ e $37,5 \%$, respectivamente). Comparadas à cirurgia combinada, as mulheres submetidas à cirurgia isolada apresentaram maior risco de óbito, independentemente da idade e estadiamento (HR = 7,23; 95\%CI:2,29-22,83). Em Rio Branco, Acre, a sobrevida em 5 anos para câncer de mama foi elevada. A cirurgia combinada à radioterapia foi independentemente associada ao menor risco de óbito.

Palavras-chave Sobrevida do câncer de mama, Tratamento, Amazônia 


\section{Introduction}

Breast cancer is a major public health problem worldwide, since is the most frequent cancer among women, with an estimated incidence rate of 43.3/100,000 women and a mortality rate of $12.9 / 100,000$ women $^{1}$. Yet, the magnitude of those rates may vary across the world, in such a way that in more developed countries incidence rates are higher than those in less developed countries $(74.1 / 100,000$ vs $31.3 / 100,000)$. On the other hand, mortality rates are almost similar among developed and developing countries $(14.9 / 100,000 \text { vs } 11.5 / 100,000)^{1}$. Since the 1990s, breast cancer mortality has been falling in North America, Australasia and several European countries. This reduction has been frequently attributed to the combined effect of mammography screening, and improvements in treatment and in the efficiency of health care systems ${ }^{2,3}$.

Long-term prognosis for patient with breast cancer has improved significantly over the past 50 years. In developed countries, such as Spain, 5 -year survival rates estimations are greater than $80 \% 4$. This progress could be explained by the combination of developments with enhanced treatments and earlier diagnosis ${ }^{5}$. Concerning to developing countries like India, the estimations indicate that 5-year survival is approximately $77 \% \%^{6}$, which is similar to Latin America countries, such as Porto Rico $(71,2 \%)^{7}$. However, health care in Latin America has been improving over the past 5 to 10 years, driven by reforms toward more universal health care access ${ }^{8}$.

Hospital-based breast cancer-specific survival studies developed in southern and southeastern Brazil showed an increased 5-year survival rates varying from $75 \%$ in Rio de Janeiro, $\mathrm{RJ}^{9}$ to $87.7 \%$ in Santa Maria, $\mathrm{RS}^{10,11}$. Classical prognostic factors such as age $(<50 />50 \text { years })^{10,11}$, stage (III-IV vs I-II $)^{9-11}$, tumor size $(\mathrm{T} 2-3 \text { vs T1 })^{8}$, number of node ${ }^{9-11}$, imunohistochemical markers (HER-2, ER and PR receptors, and p53) ${ }^{11}$, and treatment (surgery, radiation, chemotherapy, Immunotherapy, and combinations of each $)^{9,11}$, were statistically associated with breast cancer survival in Brazilian studies. There are few hospital-based breast cancer-survival studies in less developed areas in Brazil. The only study found, was carried out among 58 breast cancer patients from an oncology outpatient clinic in Maranhão ${ }^{12}$, treated with Tamoxifen, and analyzed only associations of CYP2D6 ${ }^{\star} 3,{ }^{\star} 4$, and ${ }^{\star} 10$ alleles enzyme activity with disease-free survival.
Population-based statistics on cancer incidence currently available for northern municipalities come from Belem (Pará state) and Manaus (Amazon state), and estimates for western Amazon were inexistent until 2012, when Nakashima et al. ${ }^{13}$ estimated the incidence rate for Rio Branco, state of Acre, which is located in the western Amazon. Incidence rates were estimated based on records from all hospital system units, all pathological anatomy laboratory records and the Hospital Cancer Registry database in the city of Rio Branco. In such city, age-adjusted incidence rate for breast cancer reached 41.5/100,000 wom$\mathrm{en}^{13}$. Also, age-adjusted mortality rate increased from $2.9 / 100,000$ women in 1993 to $6.4 / 100,000$ women in $2004^{14}$.

In June 2007 a High Complexity Treatment Unit (UNACON) was organized by the National Cancer Institute aiming to provide a standardized diagnosis, treatment and follow-up protocol to the population of Rio Branco. Thus, after 6 years of UNACON organization, the present study aims to estimate the hospital-based breast cancer survival of women treated in the city of Rio Branco, Acre, and predictive factors involved.

\section{Material and methods}

\section{Study population and design}

A retrospective study was carried out on a cohort of all women diagnosed with breast cancer from $1^{\text {st }}$ June 2007 to $31^{\text {st }}$ May 2012 in the UNACON, which is the only cancer center of state of Acre, located in the city of Rio Branco, western Amazon. Women of all ages treated in the UNACON of Rio Branco were eligible for the study. From the original cohort of 268 patients diagnosed with breast cancer identified on the database during the study period, 99 (36.9\%) were not followed in Rio Branco and 8 (3\%) had a second primary tumor site. Thus, 161 women were included in the study. According to the National Resolution for Human Research in Brazil (Resolução 196/96, revised by Resolução 466/12) once the present study was based on retrospective data collection, no informed consent was required. However, we obtained official approval from the Majors of the Hospitals, laboratories, Municipal and State Health secretariat to access databases, laboratory and medical records. This study was approved by the Ethical Committee of Federal University of Acre. 


\section{Collection data}

Epidemiological, clinical, pathological and molecular data were obtained from medical reports of UNACON/Acre of all breast cancer cases diagnosed from June 2007 to May 2012. All pathological anatomy laboratory records (three private and one public laboratory) were used to validate the pathological diagnosis. Patients were followed until $31^{\text {st }}$ December 2013 according to the last status (alive, death or loss of follow-up) confirmed by linkage with the death certificates from National Mortality Information System (SIM).

Morphological and topographic classification used for breast cancer was ICD-O ${ }^{15}$ and ICD- $10^{16}$, respectively. Incident cancer cases included all patients with histopathology diagnosis of cancer in the study period (ICD-10: C50 and D05.1) corresponding to malignant behavior tumors, primary site (ICD-O: code 3 ) and malignant, metastatic location (ICD-O: code 6). Follow-up information is updated every time women come to the hospital and/or take a health test in one of the laboratories of the city. Information on date and cause of death is obtained periodically through a record linkage procedure between the hospital cancer registry and the Mortality Information System (SIM) available at Rio Branco Municipal Health Secretariat and Acre State Health Secretariat. The ones that died outside Acre were identified in the National Mortality Information System.

\section{Variable}

Age at diagnosis was categorized into two groups ( $<40 / \geq 40$ years), according to the mammography screening recommendation of the Brazilian Society for Breast Cancer - SBM ${ }^{17}$. Skin color (white / non-white), marriage status (with a partner / no partner), tobacco smoking (non-smoker / current smoker), alcohol consumption (never drinker / current drinker), comorbidity (yes / no), second primary site cancer (yes / no) and family history of cancer (breast cancer and / or other cancer), and number of relatives with cancer $(<2 / \geq 2$ relatives $)$ were collected from the medical records.

Tumor stage at diagnosis was classified according to the version of the pathological TNM classification used in the year of diagnosis $(0, \mathrm{I}, \mathrm{II}$, III and IV stages). Women who received neoadjuvant therapy and women who had no surgery tumor staging were based on TNM clinical clas- sification. Tumor histology (ductal in situ/ductal invasive / other), nodal status $(\leq 2$ nodes $/>2$ nodes), size $(<2.5 \mathrm{~cm} / \geq 2.5 \mathrm{~cm})$, and grade $(1-$ well differentiated / 2-moderately well differentiated / 3-poorly differentiated / undifferentiated), margins (clear/involved) were also recorded. Information on receptor expression of cases was obtained from record review of immunohistochemistry (IHC) pathology report. According to the staining intensity, the pathologist categorized HER-2 status as positive (IHC score $=3+$ ), negative (IHC score $=0,1+$ ) or equivocal (inconclusive IHC score $=2+$ ). Estrogen receptor expression (ER) and progesterone receptor expression (PR) were classified as positive / negative. Later, $\mathrm{ER}$ and PR variables were categorized into combined hormone receptors expression $(\mathrm{ER}+\mathrm{PR}+$ or ER+PR- / ER-PR+ / ER-PR-). The first course of treatment was classified as chemotherapy associated with surgery (including breast-conserving and mastectomy in addition to sentinel node biopsy or complete axillary dissection) and radiotherapy, surgery alone and surgery associated with radiotherapy.

\section{Statistical analysis}

The chi-square method was used to test the differences in the proportions among categorical variables with a significance level of $5 \%$. In order to proceed the survival analyses, three cohort periods were defined according to the year of diagnosis and follow-up time: 2007-2012 (1year follow-up), 2007-2011 (2-years follow-up), 2007-2008 (5-years follow-up). Survival time was defined as the length of time (months) from the diagnostic date to date of death of breast cancer (failure). Patients who were still alive at the end of the study were censored in the cohort; those patients who died from other causes but breast cancer were censored on the date of death; and all those who moved from the city were censored on the date of last appointment in the hospital.

One-, 2- and 5-year breast cancer-specific survival rates were estimated using Kaplan-Meier method. Differences between Kaplan-Meier curves were assessed with the Log-Rank test ${ }^{18}$. Breast cancer-specific survival rates, stratified by age ( $<40$ years, $\geq 40$ years) and stage (I-II, III), were estimated by Kaplan-Meier method. Survival curves were evaluated by using a Log-Rank test and 95\% confidence interval (95\% CI) for each stratum. Adjusted hazard ratios (HR) and 95\% confidence interval (95\% CI) were estimated by proportional Cox regression models ${ }^{19}$. Maximum 
$\log$ likelihood was used to estimate the coefficient model, partial and global $\mathrm{R}^{2}$ were used to evaluate the goodness of fit models, while log minus log curves were used to test the assumption of proportional risks over time.

In order to reduce the loss of power in the present study, only variables with less than $20 \%$ of missing values were included in the stratified survival analyses and Cox regression model. All analyses were performed by using SPSS statistical software, version 17.0. Statistical significance level was set at 0.05 for two-sided tests.

\section{Results}

From 161 cases, 44 (20.5\%) were dead from breast cancer. Median follow-up time of patients at the closing date was $12( \pm 2.61)$ months for the 2007-2012 cohort, 24 ( \pm 6.1) months 2007-2011 cohort and $57( \pm 16.2)$ months for 2007-2008 cohort. The mean age was $52.8( \pm 13$, ranging from 21 to 87 years old) and the median was 52.5 years old. Table 1 shows that 1-, 2-, and 5-year overall breast cancer survival were $95.5 \%, 83.7 \%$, and $87.3 \%$ respectively, but no statistical significance was found among the survival curves. Statistically, poorer 5-year survival was observed for wom- en with two or more family history of cancer, compared to those with less than two relatives ( $60 \%$ vs $94.7 \%$; LR: $95 \%=0.012$ ).

Fifty-eight percent of patients were early stage at diagnosis and stage at diagnosis was inversely related to 1-, 2-, and 5-year survival (Table 1). According to Table 1, women at early stage (stage 0 -II) had 1-, 2- and 5-year survival of respectively $98.9 \%, 92.2 \%$, and $93.3 \%$. Women at stage-III had respectively $94.3 \%, 76.8 \%$ and $80.7 \%$ in 1 -, 2 -, and 5-year survival, while women at stage IV had $75 \%, 21.4 \%$ and $50 \%$ respectively. Two-year survival was statistically higher for women presenting tumor size smaller than $2.5 \mathrm{~cm}$ (90.4\%), as compared to women presenting tumor size of $2.5 \mathrm{~cm}$ and plus (67.7\%). Positive margins significantly worsen 2 - and 5-year survival (73.4\% and $77.8 \%$, respectively) as compared to negative margins (91.7\% and $97.6 \%$ respectively). Positive progesterone receptor increased 1-, 2-, and 5 - year survival as compared to negative receptor (LR: $95 \%=0.042,0.014,0.042$, respectively). Although positive estrogen receptor influenced only 5 -year breast cancer-specific survival (96.3\% vs $70 \%$ ), when combining both positive progesterone and estrogen receptor, 1-, 2-, and 5- survival were statistically higher comparing to any other hormone receptor combination, al-

Table 1. Breast cancer survival rates according to sociodemographic, clinical, histopathological and molecular characteristics and treatment. Rio Branco, Brazil (2007-2012)

\begin{tabular}{|c|c|c|c|c|c|c|c|c|c|}
\hline \multirow{3}{*}{ Variable } & \multicolumn{6}{|c|}{ Overall $^{\mathrm{a}}$ / death $(\%)$} & \multicolumn{3}{|c|}{ Specific Survival (\%) } \\
\hline & \multicolumn{2}{|c|}{1 year } & \multicolumn{2}{|c|}{2 years } & \multicolumn{2}{|c|}{5 years } & \multirow{2}{*}{$\begin{array}{r}\text { year } \\
95.5\end{array}$} & \multirow{2}{*}{$\begin{array}{r}2 \text { years } \\
83.7\end{array}$} & \multirow{2}{*}{\begin{tabular}{|r|}
5 years \\
87.3
\end{tabular}} \\
\hline & 161 & $07(4.3)$ & 145 & $22(15.2)$ & 52 & $06(11.5)$ & & & \\
\hline \multicolumn{10}{|l|}{ Age at diagnosis } \\
\hline$<40$ years & 23 & $0(0.0)$ & 20 & $04(20.0)$ & 09 & $02(22.2)$ & 100 & 100.0 & 75.0 \\
\hline$\geq 40$ years & 138 & $07(5.1)$ & 125 & $18(14.4)$ & 43 & $04(9.3)$ & 94.8 & 84.6 & 89.9 \\
\hline \multicolumn{10}{|l|}{ Marriage status } \\
\hline no partner & 78 & $03(3.8)$ & 72 & $10(13.9)$ & 24 & $04(16.7)$ & 96.1 & 85.1 & $82.6^{*}$ \\
\hline with a partner & 68 & $01(1.5)$ & 60 & $07(11.7)$ & 22 & $0(0.0)$ & 98.5 & 87.5 & 100 \\
\hline \multicolumn{10}{|l|}{ Skin color } \\
\hline white & 26 & $0(0.0)$ & 22 & $1(4.5)$ & 10 & $02(20.0)$ & 100 & 95.0 & 80.0 \\
\hline non-white & 107 & $04(3.7)$ & 97 & $15(15.5)$ & 29 & $02(6.9)$ & 96.2 & 83.3 & 92.6 \\
\hline \multicolumn{10}{|l|}{ Relatives with cancer } \\
\hline$<2$ relatives & 135 & $04(3.0)$ & 122 & $14(11.5)$ & 41 & $02(4.9)$ & 97.0 & 87.8 & 94.7 \\
\hline$\geq 2$ relatives & 14 & $0(0.0)$ & 11 & $3(27.3)$ & 05 & $02(40.0)$ & 100 & 70.0 & $60.0^{*}$ \\
\hline \multicolumn{10}{|l|}{ Stage at diagnosis } \\
\hline 0-II stage & 94 & $01(1.1)$ & 82 & $06(7.3)$ & 31 & $02(6.6)$ & 98.9 & 92.2 & 93.3 \\
\hline III stage & 54 & $03(5.6)$ & 51 & $11(21.6)$ & 17 & 03(17.4) & $94.3^{* *}$ & $76.8^{\star *}$ & $80.7^{\star *}$ \\
\hline IV stage & 09 & $02(22.2)$ & 08 & $04(50.0)$ & 04 & $01(25.0)$ & $75.0^{\star *}$ & $21.4^{\star *}$ & $50.0^{\star *}$ \\
\hline
\end{tabular}


Table 1. Breast cancer survival rates according to sociodemographic, clinical, histopathological and molecular characteristics and treatment. Rio Branco, Brazil (2007-2012)

\begin{tabular}{|c|c|c|c|c|c|c|c|c|c|}
\hline \multirow{2}{*}{ Variable } & \multicolumn{6}{|c|}{ Overall $^{\mathrm{a}}$ / death $(\%)$} & \multicolumn{3}{|c|}{ Specific Survival (\%) } \\
\hline & \multicolumn{2}{|c|}{1 year } & \multicolumn{2}{|c|}{2 years } & \multicolumn{2}{|r|}{5 years } & \multirow[t]{2}{*}{1 year } & \multirow[t]{2}{*}{2 years } & \multirow[t]{2}{*}{5 years } \\
\hline Histologic grades & & & & & & & & & \\
\hline 1 grade & 14 & $0(0.0)$ & 13 & $01(7.7)$ & 07 & $0(0.0)$ & 100 & 92.3 & 100.0 \\
\hline 2-3 grade & 13 & $06(4.3)$ & 123 & $17(13.8)$ & 41 & $05(12.2)$ & 95.6 & 85.3 & 86.7 \\
\hline \multicolumn{10}{|l|}{ Tumor size } \\
\hline$<2.5 \mathrm{~cm}$ & 122 & $04(3.3)$ & 110 & $10(9.1)$ & 38 & $03(7.9)$ & 96.7 & 90.4 & 91.7 \\
\hline$\geq 2.5 \mathrm{~cm}$ & 22 & $01(4.5)$ & 19 & $06(31.6)$ & 08 & $01(12.5)$ & 95.5 & 67.7 & 87.5 \\
\hline \multicolumn{10}{|l|}{ Histological types } \\
\hline ductal in situ & 13 & $0(0.0)$ & 12 & $01(8.3)$ & 03 & $0(0.0)$ & 100 & 91.7 & 100.0 \\
\hline ductal invasive & 129 & $06(4.7)$ & 117 & $16(13.7)$ & 43 & $05(11.6)$ & 95.2 & 85.3 & 87.7 \\
\hline others & 13 & $0(0.0)$ & 10 & $01(10.0)$ & 03 & $0(0.0)$ & 100 & 88.9 & 100.0 \\
\hline \multicolumn{10}{|l|}{ Axillar node dissection } \\
\hline no & 23 & $01(4.3)$ & 20 & $01(5.0)$ & 07 & $0(0.0)$ & 95.5 & 94.7 & 100.0 \\
\hline yes & 107 & $02(1.9)$ & 96 & $11(11.5)$ & 33 & $04(12.1)$ & 98.1 & 87.9 & 87.1 \\
\hline \multicolumn{10}{|l|}{ Positive nodes } \\
\hline$\leq 2$ nodes & 66 & $01(1.5)$ & 58 & $04(6.9)$ & 25 & $04(16.0)$ & 98.5 & 93.0 & 83.3 \\
\hline$>2$ nodes & 41 & $01(2.4)$ & 38 & $07(18.4)$ & 07 & $02(7.4)$ & 97.6 & 80.1 & 92.0 \\
\hline \multicolumn{10}{|l|}{ Margins status } \\
\hline negative & 100 & $02(2.0)$ & 88 & $07(8.0)$ & 32 & $01(3.1)$ & 98.0 & 91.7 & 97.6 \\
\hline positive & 24 & $01(4.2)$ & 23 & $06(26.1)$ & 09 & $02(22.2)$ & 95.8 & $73.4^{\star}$ & $77.8^{\times}$ \\
\hline \multicolumn{10}{|l|}{ Estrogen receptor } \\
\hline positive & 98 & $04(4.1)$ & 88 & $09(10.2)$ & 29 & $01(3.4)$ & 95.8 & 89.1 & 96.3 \\
\hline negative & 46 & $02(4.3)$ & 42 & $09(21.4)$ & 15 & $04(26.7)$ & 95.5 & 76.7 & $70.0^{\star}$ \\
\hline \multicolumn{10}{|l|}{ Progesterone receptor } \\
\hline positive & 81 & $01(1.2)$ & 75 & $06(8.0)$ & 26 & $01(3.8)$ & 98.7 & 91.4 & 95.7 \\
\hline negative & 61 & $05(8.2)$ & 53 & $12(22.6)$ & 16 & $04(25.0)$ & $91.6^{*}$ & $75.1^{\star}$ & $73.7^{\star}$ \\
\hline \multicolumn{10}{|l|}{ Tumor marker } \\
\hline $\mathrm{ER}+\mathrm{PR}+$ & 78 & $01(1.3)$ & 72 & $06(8.3)$ & 24 & $01(4.2)$ & 98.7 & 91.0 & 95.5 \\
\hline ER+PR-/ER-PR+/ER-PR- & 63 & $05(7.9)$ & 55 & $12(21.8)$ & 17 & $04(23.5)$ & $91.8^{*}$ & $76.1^{\star}$ & $73.9^{\star}$ \\
\hline \multicolumn{10}{|l|}{ Status HER2 } \\
\hline positive & 30 & $01(3.3)$ & 27 & $04(14.8)$ & 09 & $01(11.1)$ & 96.7 & 84.3 & 85.7 \\
\hline negative & 91 & $05(5.5)$ & 81 & $13(16.0)$ & 21 & $04(19.4)$ & 94.4 & 83.0 & 80.2 \\
\hline \multicolumn{10}{|l|}{ Treatment } \\
\hline surgery and radiotherapy ${ }^{\mathrm{b}}$ & 96 & $01(1.0)$ & 88 & $05(5.7)$ & 33 & $03(9.1)$ & 99.0 & 94.0 & 90.6 \\
\hline surgery $^{c}$ & 45 & $02(4.4)$ & 39 & $11(28.2)$ & 12 & $01(8.3)$ & $95.5^{\star *}$ & $71.0^{* *}$ & $91.7^{\star}$ \\
\hline others $^{\mathrm{d}}$ & 09 & $02(22.2)$ & 07 & $03(42.9)$ & 04 & $02(50.0)$ & $77.8^{\star *}$ & $57.1^{\star *}$ & $37.5^{\star}$ \\
\hline
\end{tabular}

${ }^{\mathrm{a}}$ Total may change due to missing values; ${ }^{\mathrm{b}}$ Surgery associated with radiotherapy only (or also combined with chemotherapy and/ or hormone therapy); ${ }^{\mathrm{c}}$ Surgery only (or also combined with chemotherapy and/or hormone therapy); ${ }^{\mathrm{d}}$ Radiotherapy and/or chemotherapy and/or hormone therapy; ${ }^{*} \log$-Rank $95 \% \mathrm{CI}$ (p-value) $<0,05 ;{ }^{* *} \log$-Rank $95 \% \mathrm{CI}$ (p-value) $<0,01$.

though borderline significance was observed in 1- and 5-year survival. Negative HER-2 receptor was more frequent than positive HER-2 receptor (HER-2+ $(\mathrm{n}=30 ; 18.6 \%)$; HER-2 - $(\mathrm{n}=$ 91; 56.5\%); missing $(\mathrm{n}=11 ; 24.8 \%))$. There was no inconclusive result for HER-2 in IHC test in our study population. Surgery combined to ra- diotherapy treatment influenced significantly $1-$, 2 -, and 5-year survival $(99 \%, 94 \%$, and $90.6 \%$, respectively) as compared to other treatments (77\%, $57.1 \%$, and $37.5 \%$, respectively).

As stratified by age $(<40$ years old $/ \geq 40$ years old), positive progesterone receptor significantly increased 1- and 2-year survival (S(1):98.6\% vs 
90.5\%; $\mathrm{S}(2): 93.5 \%$ vs $73.2 \%)$ within old women, but not in young women (Figure 1). Similar results were found for both positive estrogen and progesterone receptor among women with 40 years and older $(\mathrm{S}(1): 98.5 \%$ vs $90.4 \%, 95 \% \mathrm{LR}$ $=0.049 ; \mathrm{S}(2): 93.1 \%$ vs $74.5 \%, 95 \% \mathrm{LR}=0.008)$, comparing to any other hormonal receptor combination (Figure 1). Over 2 lymph nodes involved seemed to influence 2-year breast cancer survival among women under 40 years old, with a borderline significance $(95 \% \mathrm{LR}=0.065)$.

Lymph node involvement (> 2 lymph node) significantly decreased 2-year breast cancer survival within early stage patients $(S(2): 97.7 \%$ vs 75\%, 95\% LR: 0.021) but not late stage patients (Table 2). On the other hand, positive surgical

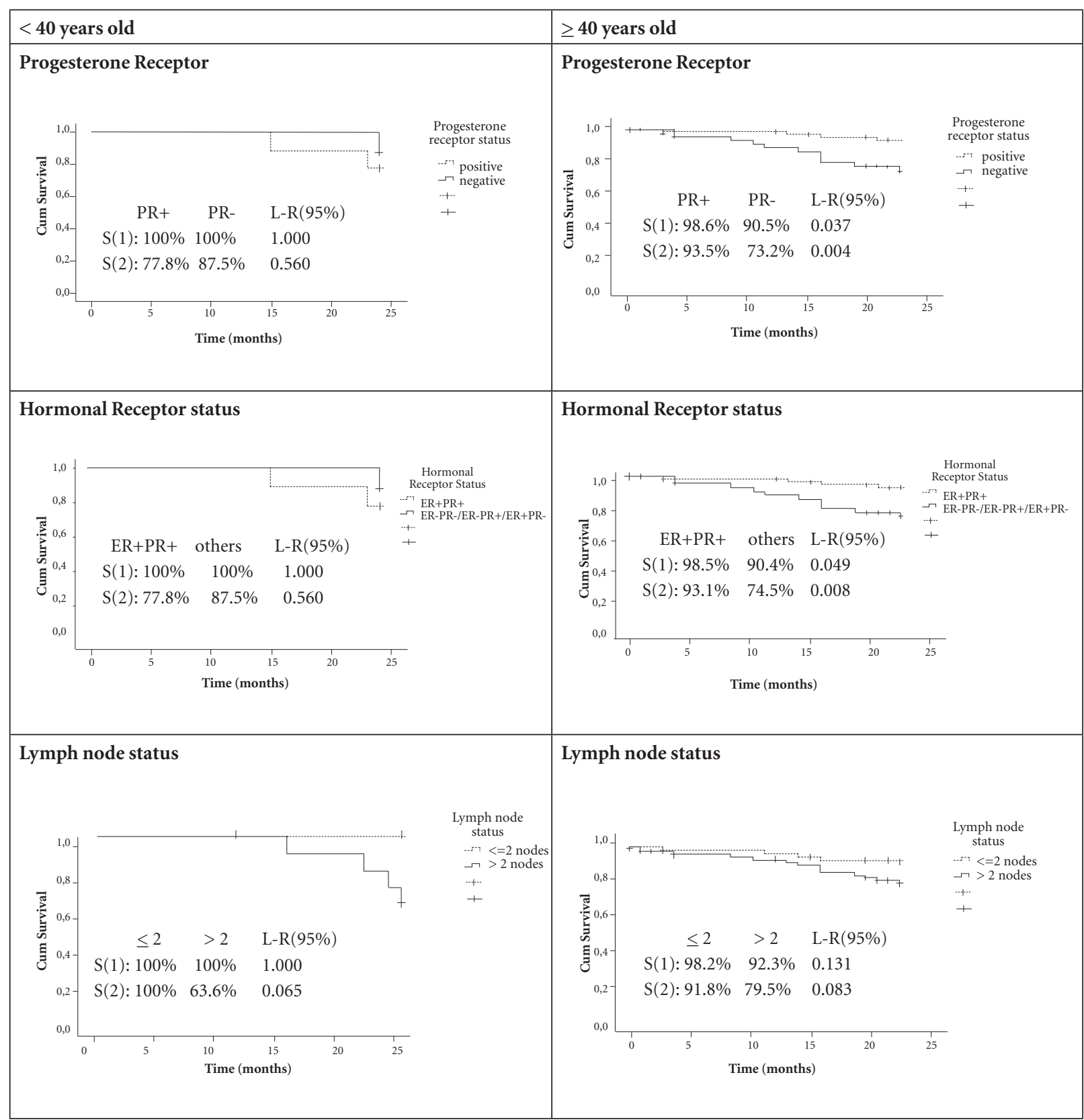

Figure 1 Survival curves for progesterone receptor, hormonal receptor status and lymph node status, according to age group (161 breast cancer patients, western Amazon, Brazil). 
Table 2. Breast cancer survival rates according to clinical, histopathological, molecular and treatment, stratified by stage at diagnosis. Rio Branco, Brazil (2007-2012).

\begin{tabular}{|c|c|c|c|c|c|c|c|c|c|c|c|c|c|}
\hline \multirow{3}{*}{ Variable } & \multicolumn{8}{|c|}{ 0-II Stage } & \multicolumn{5}{|c|}{ III Stage } \\
\hline & \multicolumn{4}{|c|}{1 year } & \multicolumn{4}{|c|}{2 years } & \multicolumn{4}{|c|}{1 year } & \multirow{2}{*}{$\begin{array}{c}2 \text { years } \\
S(2)\end{array}$} \\
\hline & $\mathbf{n}^{\mathrm{a}}$ & death & $S(1)$ & $\mathbf{n}^{\mathrm{a}}$ & death & $S(2)$ & $\mathbf{n}^{\mathrm{a}}$ & death & $S(1)$ & $\begin{array}{c}\text { Log Rank } \\
95 \%\end{array}$ & $\mathbf{n}^{\mathrm{a}}$ & death & \\
\hline \multicolumn{14}{|l|}{ Age at diagnosis } \\
\hline$<40$ years & 13 & $0(0.0)$ & 100 & 11 & $01(9.1)$ & 90.9 & 08 & $0(0.0)$ & 100 & 0.460 & 07 & $03(42.9)$ & 57.1 \\
\hline$\geq 40$ years & 81 & $01(1.2)$ & 98.8 & 71 & $05(7.0)$ & 92.5 & 46 & $03(6.5)$ & 93.3 & & 44 & $08(18.2)$ & 80.6 \\
\hline \multicolumn{14}{|l|}{ Tumor size } \\
\hline$<2.5 \mathrm{~cm}$ & 81 & $01(1.2)$ & 98.8 & 71 & $04(5.6)$ & 94.0 & 36 & $01(2.8)$ & 97.2 & 0.421 & 35 & $05(14.3)$ & 84.8 \\
\hline$\geq 2.5 \mathrm{~cm}$ & 10 & $0(0.0)$ & 100 & 09 & $02(22.2)$ & 76.2 & 12 & $01(8.3)$ & 91.7 & & 10 & $04(40.0)$ & 60.0 \\
\hline \multicolumn{14}{|l|}{ Axillar node dissection } \\
\hline no & 19 & $0(0.0)$ & 100 & 17 & $01(5.9)$ & 94.1 & 01 & $0(0.0)$ & 100 & 0.884 & 01 & $0(0.0)$ & 100 \\
\hline yes & 60 & $01(1.7)$ & 98.3 & 52 & $03(5.8)$ & 93.7 & 47 & $01(2.1)$ & 97.9 & & 44 & $08(18.2)$ & 80.9 \\
\hline \multicolumn{14}{|l|}{ Positive nodes } \\
\hline$\leq 2$ nodes & 52 & $01(1.9)$ & 98.1 & 44 & $01(2.3)$ & 97.7 & 14 & $0(0.0)$ & 100 & 0.515 & 14 & $03(21.4)$ & 78.6 \\
\hline$>2$ nodes & 08 & $0(0.0)$ & 100 & 08 & $02(25.0)$ & $75.0^{* *}$ & 33 & $01(3.0)$ & 97.0 & & 30 & $05(16.7)$ & 82.0 \\
\hline \multicolumn{14}{|l|}{ Margin } \\
\hline negative & 63 & $01(1.6)$ & 98.4 & 53 & 01(1.9) & 98.1 & 37 & $01(2.7)$ & 97.3 & 0.192 & 35 & $06(17.1)$ & 82.0 \\
\hline positive & 17 & $0(0.0)$ & 100 & 17 & 03(17.6) & $82.4^{* *}$ & 07 & $01(14.3)$ & 85.7 & & 06 & $03(50.0)$ & $50.0^{\star}$ \\
\hline \multicolumn{14}{|l|}{ Estrogen receptor } \\
\hline positive & 61 & $01(1.6)$ & 98.4 & 53 & $04(7.5)$ & 92.1 & 29 & $01(3.4)$ & 96.6 & 0.365 & 28 & $03(10.7)$ & 88.5 \\
\hline negative & 24 & $0(0.0)$ & 100 & 21 & $02(9.5)$ & 88.9 & 21 & $02(9.5)$ & 89.7 & & 20 & $06(30.0)$ & 66.9 \\
\hline \multicolumn{14}{|l|}{ Progesterone receptor } \\
\hline positive & 50 & $01(2.0)$ & 98.0 & 45 & $03(6.7)$ & 93.2 & 25 & $0(0.0)$ & 100 & 0.058 & 24 & $02(8.3)$ & 90.7 \\
\hline negative & 35 & $0(0.0)$ & 100 & 29 & $03(10.3)$ & 87.2 & 23 & $03(13.0)$ & 86.3 & & 22 & $07(31.8)$ & $65.5^{\star}$ \\
\hline \multicolumn{14}{|l|}{ Hormonal status } \\
\hline $\mathrm{ER}+\mathrm{PR}+$ & 48 & $01(2.1)$ & 97.9 & 43 & $03(7.0)$ & 92.8 & 24 & $0(0.0)$ & 100 & 0.070 & 23 & $02(8.7)$ & 90.2 \\
\hline ER+PR-/ER-PR+/ ER-PR- & 36 & $0(0.0)$ & 100 & 30 & $03(10.0)$ & 87.8 & 24 & $03(12.5)$ & 86.9 & & 23 & $07(30.4)$ & $67.1^{\star}$ \\
\hline \multicolumn{14}{|l|}{ Treatment } \\
\hline Surgery and radiotherapy ${ }^{\mathrm{b}}$ & 56 & $0(0.0)$ & 100 & 51 & $02(3.9)$ & 95.6 & 38 & $01(2.6)$ & 97.4 & 0.529 & 35 & $03(8.6)$ & 91.2 \\
\hline Surgery ${ }^{c}$ only & 34 & $01(2.9)$ & 97.1 & 28 & $03(10.7)$ & 89.0 & 09 & $01(11.1)$ & 88.9 & & 09 & $06(66.7)$ & 33.3 \\
\hline Others $^{\mathrm{d}}$ & 04 & $0(0.0)$ & 100 & 03 & $01(33.3)$ & 66.7 & 01 & $0(0.0)$ & 100 & & 01 & $0(0.0)$ & 100 \\
\hline
\end{tabular}

margins significantly reduced 2-years survival within both early stage ( $98.1 \%$ vs $82.4 \%, 95 \%$ LR $=0.024)$ and stage-III patients ( $82 \%$ vs $50 \%, 95 \%$ $\mathrm{LR}=0.040)$. Among stage-III patients, positive progesterone receptor $(90.7 \%$ vs $65.5 \%, 95 \%$ LR $=0.029$ ), combining positive estrogen and progesterone receptors $(90.2 \%$ vs $67.1 \%, 95 \% \mathrm{LR}=$ $0.045)$, and surgery associated to radiotherapy treatment ( $91.2 \%$ vs $33.3 \%$, 95\% LR < 0.001) significantly increased 2-year breast cancer survival.

According to Table 3, adjusting by age, stageIII significantly increased the risk of death in over 3 -fold as compared to early stage at diag- nosis ( $\mathrm{HR}=3.37$; 95\% CI: 1.24-9.18). Similarly, tumor size $\geq 2.5 \mathrm{~cm}(\mathrm{HR}=3.39$; 95\% CI: 1.18 9.71) and positive surgical margins ( $\mathrm{HR}=5.12$; 95\% CI: 1.65-15.86) significantly increased the risk of death in this population, regardless of age and stage. Women presenting negative progesterone receptors had a risk 3-fold higher of dying of breast cancer, comparing to the positive ones. Women with negative estrogen receptor presented a risk of death in over 2-foldas compared to women with positive estrogen receptor. However, no statistical significance was observed (95\% CI: 0.83-6.61). 
Table 3. Crude and adjusted hazard ratios estimates for breast cancer death among women from Rio Branco/AC, Brazil (2007-2012).

\begin{tabular}{|c|c|c|c|c|c|c|}
\hline Variable & $\begin{array}{c}\text { Crude } \\
\text { HR }\end{array}$ & $95 \% \mathrm{CI}$ & $\mathrm{HR}^{\mathrm{a}}$ & $95 \% \mathrm{CI}$ & $\mathrm{HR}^{\mathrm{b}}$ & $95 \% \mathrm{CI}$ \\
\hline \multicolumn{7}{|l|}{ Stage at diagnosis } \\
\hline 0-II & 1 & - & 1 & - & 1 & - \\
\hline III & 3,46 & $1.27-9.36$ & 3.37 & $1.24-9.18$ & 3.37 & $1.24-9.18$ \\
\hline \multicolumn{7}{|l|}{ Tumor size } \\
\hline$<2.5 \mathrm{~cm}$ & 1 & - & 1 & - & 1 & - \\
\hline$\geq 2.5 \mathrm{~cm}$ & 3.65 & $1.32-10.06$ & 3.63 & $1.31-10.00$ & 3.39 & $1.18-9.71$ \\
\hline \multicolumn{7}{|l|}{ Positive nodes } \\
\hline$\leq 2$ nodes & 1 & - & 1 & - & 1 & - \\
\hline$>2$ nodes & 2.74 & $0,80-9.37$ & 2.65 & $0.76-9.21$ & 1.64 & $0.40-6.73$ \\
\hline \multicolumn{7}{|l|}{ Margin } \\
\hline negative & 1 & - & 1 & - & 1 & - \\
\hline positive & 3.35 & $1.12-9.98$ & 3.35 & $1.12-9.97$ & 5.12 & $1.65-15.86$ \\
\hline \multicolumn{7}{|l|}{ Estrogen receptor } \\
\hline Positive & 1 & - & 1 & - & 1 & - \\
\hline Negative & 2.18 & $0.86-5.50$ & 2.25 & $0.88-5.75$ & 2.35 & $0.83-6.61$ \\
\hline \multicolumn{7}{|l|}{ Progesterone receptor } \\
\hline Positive & 1 & - & 1 & - & 1 & - \\
\hline Negative & 3.17 & $1.19-8.47$ & 3.22 & $1.20-8.63$ & 3.05 & $1.03-9.04$ \\
\hline \multicolumn{7}{|l|}{ Hormone status } \\
\hline $\mathrm{ER}+\mathrm{PR}+$ & 1 & - & 1 & - & 1 & - \\
\hline ER+ PR-/ER-PR+/ER-PR- & 2.89 & $1.08-7.72$ & 2.96 & $1.09-7.97$ & 2.79 & $0.93-8.31$ \\
\hline \multicolumn{7}{|l|}{ Treatment } \\
\hline surgery and radiotherapy ${ }^{c}$ & 1 & - & 1 & - & 1 & - \\
\hline surgery $^{\mathrm{d}}$ & 5.66 & $1.96-16.32$ & 5.74 & $1.96-16.80$ & 7.23 & $2.29-22.83$ \\
\hline others ${ }^{\mathrm{e}}$ & 8.74 & $2.08-36.61$ & 8.97 & 2.06-38.98 & 6.63 & $0.69-63.30$ \\
\hline
\end{tabular}

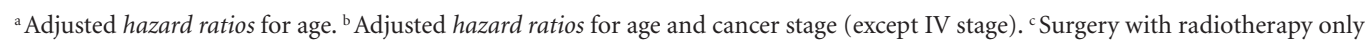
(or also combined with chemotherapy and/or hormone therapy) ${ }^{\mathrm{d}}$ Surgery only (or also combined with chemotherapy and/or hormone therapy). ${ }^{e}$ Radiotherapy and/or chemotherapy and/or hormone therapy.

Comparing to surgery associated with radiotherapy treatment, women who had undergone surgery (alone or combined to chemotherapy) were at higher risk of death, regardless of age and stage (HR $=7.23$; 95\% CI: 2.29-22.83). Increased risk of death was observed for those under other treatments (radiotherapy alone / with chemotherapy and/or hormone therapy, chemotherapy alone / with hormone therapy), although no significance was observed $(\mathrm{HR}=6.63 ; 95 \% \mathrm{CI}$ : 0.69-63.30).

\section{Discussion}

In the present study, 5-year breast cancer-specific survival rate was $87.3 \%$, which is similar to those found in European countries such as Spain $(\geq$
$80 \%)^{4}$, and even higher than those found in developing countries such as India $(77 \%)^{6}$ and Porto Rico $(71,2 \%)^{7}$. Compared to more developed areas in Brazil, our finding was similar to the survival rate found in Santa Maria, SC, $(87.7 \%)^{10}$; and even higher than those found in Belo Horizonte $(78.5 \%)^{19}$, Florianópolis $(76.2 \%)^{11}$ and Barretos $(74.8 \%)^{20}$. However, the difference in breast cancer survival rate among countries and areas of the same country exist and are not easy to interpret. The highest survival rate in one country versus another may be due to either availability of better treatment, or similar treatment being more effective because diagnosis is made at an earlier stage at disease ${ }^{21}$.

Surprisingly, we observed that almost twothird of breast cancer observed in the present study $(58.4 \%)$ was in early stage (0-II) at diagno- 
sis, which is very similar to the frequencies found in developed countries and more developed areas in Brazil ${ }^{10,11,19,20}$. Late stage at diagnosis has been independently associated with poorer survival rates worldwide and in Brazil $1^{4,5,10,11,19,20,22}$. In a cohort study including 252 patients in Santa Maria (South of Brazil) 5-year breast cancer-survival rate was $97 \%$ for stage-I, $87 \%$ for stage-II, $73 \%$ for stage-III, and $57 \%$ for stage $\mathrm{IV}^{10}$. The largest study in Brazil evaluated 5,257 breast cancer patients from a public hospital cancer registry in Barretos, $\mathrm{SP}^{20}$. According to this study $38.9 \%$ of women were diagnosed at stage-II, while $37 \%$ were diagnosed at stage III-VI. Similar to Moraes`s study, 5-year breast cancer-specific survival rate was $95.2 \%$ for stage-I, $87.1 \%$ for stage-II, and $58.4 \%$ for stage III-IV ${ }^{20}$. Corroborating with previous studies, late stage at diagnosis $(34,4 \%$ stage-III), and statistically significant inverse relation between 5 -year breast cancer survival rate and stage at diagnosis were observed in our study, which were similar to those found in more developed areas of the country. These 5-year survival rates are even higher than those found in Thailand (stage-I: $86 \%$, II: $68 \%$, III: $37 \%$, and IV: $21 \%)^{23}$ and Spain (I: $97 \%$, II: $88 \%$, III: $70.1 \%$, IV: $24.5 \%)^{4}$. However, epidemiological evidences suggest that demographic (age, skin color, and so on), clinic (lymph node, treatment, surgical margins, and so on) and immunohistochemical (hormone receptor, HER2, and so on) features influence breast cancer-specific survival rates regardless of the stage at diagnosis $\mathrm{s}^{4,19,23-29}$.

Breast cancer in women under 40 years is uncommon, accounting for approximately $7 \%$ of all cases diagnosed, and it is usually associated with poorer prognosis when compared to women over age $40^{24,25}$. However, some studies have demonstrated prognostic differences only in younger women with early-stage disease ${ }^{24,26}$. Although in the present study the absolute number of young women with breast cancer is very small $(\mathrm{n}=23)$, it represents $14,3 \%$ of all breast cancer cases diagnosed in Rio Branco from June 2007 to May 2012. Such result is higher than found in some case series from other studies where such percentage varied from $5 \%-7 \%{ }^{25}$. Also, we have found that women under 40 years presented poorer 2- and 5-year survival rate, although no statistical significance was observed. It remains unclear why young women with breast cancer have a poorer prognosis. Previous studies have suggested that young women commonly have tumors with poor prognostic features (higher histological grade, negative estrogens receptor status, HER-2 overexpression and high grade of affected lymph nodes ${ }^{24,27}$. However, when analyzing those features according to age, our results showed that among young women $(<40$ years old) only affected lymph nodes seemed to influence 2-year survival rate, with a borderline significance ( $p=0.065$, Figure- 1 ). On the other hand, among women $\geq 40$ years, those who were negative for at least one hormone receptor, and those who were negative progesterone receptor, had poorer 1- and 2-year breast cancer-specific survival rate.

Other studies have suggested that the poor survival rate could also be due to a delay in diagnosis in women under 40 years old. Firstly because this group of women is uncovered by the mammography screening program (40-59 years and/or 50-59 years); and secondly because early detection of tumor in patients under 40 years who had been submitted to screening mammography can be difficult due to higher density of mammary gland $\mathrm{s}^{30}$. In this sense, when stratified by stage, our data showed a poor 2-year survival only for young women at the late stage (stageIII). Nevertheless, Garicochea et al. ${ }^{24}$ developed a hospital-based survival study in Porto Alegre (Brazil), aiming to analyze age as a prognostic factor of early breast cancer. Authors found that patients under 40 years with clinical stage I had higher risk of disease relapse than old women. No differences were observed among women in stage-II. As in Garicochea`s study, the stage-III patients were not included and outcome evaluated was the disease relapse instead of breast cancer specific survival; comparisons with our results are limited. However, in retrospective studies of Brazilian patients developed in Santa Maria ${ }^{10}$ and Joinville $^{22}$, age was not a prognostic factor for survival, regardless of clinical stage.

Recent gene expression studies have confirmed that breast cancer encompasses four distinct biological subtypes (Luminal A, Luminal B, HER2-enriched and basal like by gene profile) with diverse etiology, therapeutic indications, and clinical outcomes ${ }^{31}$. Human epidermal growth factor 2 (HER2), estrogen (ER), and progesterone (PR) receptors are the three most common diagnostic markers that drive the clinical management of breast cancer patients ${ }^{31}$. Since certain imunohistochemical (IHC) markers can provide prognosis and predictive treatment information, and also have easy clinical accessibility, they have been used in an attempt to determine intrinsic subtypes: Luminal-A is $\mathrm{ER}+$ and/or $\mathrm{PR}+1$ HER2-, Luminal-B is ER+ and/or PR+ / HER2+, 
HER2 overexpressed is ER- / PR- / HER2+, and the triple negative is ER- / PR- / HER2- ${ }^{32}$.

Although PR is usually used along with ER to assess the patient's responsiveness to hormone therapy, some retrospective analyses of clinical cohorts have shown that PR is also useful for assessing the prognosis regardless of other factors, including $\mathrm{ER}^{33}$. However, 5-year overall survival benefit of $\mathrm{PR}+$ patients differed according to age, stage and lymph node status ${ }^{33}$. Similarly, in the present study $\mathrm{PR}+$ breast cancer patients presented better survival rate as compared to ER- patients, although, PR+ seemed to increase 1- and 2-year survival rates only among late-stage women, while no significance was found for ER+/-. A possible explanation for such findings could be the hypothesis that $\mathrm{ER}+/$ - and $\mathrm{RP}+/$ - are originated from different cell type, directing biological pathways and control metastatic processes. Thus, in healthy mammary tissue, PR+ cells do not appear to be proliferative, and proliferation seems to be mediated in a paracrine fashion ${ }^{34}$. However, in breast cancer tumors, progesterone was able to mediate the proliferation of breast cancer cells in a way that appears to be more independent of paracrine signaling ${ }^{34,35}$.

On the other hand, our results suggest that the combined effect of ER+ and PR+ presented a higher 2- and 5-year survival rate as compared to women with at least one negative hormone receptor. A plausible hypothesis is that since progesterone is not always co-expressed with ER, metastases from PR+ tumors seems to frequently lose PR expression after systemic spread and this change in the tumor phenotype is associated with a more unfavorable prognosis ${ }^{36}$.

Although surgery is accepted as a standard treatment for patients with early-stage breast cancer and is usually followed by adjuvant chemotherapy or radiotherapy to control local recurrence arising from residual disease, it remains controversial whether the risk of cardiac disease outweighs the disease-free survival benefit. In the present study, surgery combined with radiotherapy was directly related to better 1-, 2- and 5-year survival as compared to surgery with / without chemotherapy and other treatment combinations. The same pattern was observed when evaluating 1- and 2-year survival rate for treatment stratified by stage, although statistical significance was only found for 2-year survival rates within stage-III ( $p$-value: 0.001 ), probably due to the small sample size. Thus, compared to women who underwent surgery combined with radiotherapy, women who underwent to surgery with- out radiotherapy or any other treatment combination were at higher risk of death in two years, even adjusted by age and stage (Table 3 ). Recent Brazilian studies investigated breast cancer survival according to treatment, and they found out better survival rates for women under surgery only, even controlled by age and stage. However, all of them evaluated 5- and/or 10-year breast cancer survival rates; and none of them evaluated the combined effect among women under both treatments ${ }^{10,11,18,36}$.

Since breast-conserving surgery became the treatment of choice for patients with early-stage breast cancer, the role that surgical margins play is poorly understood and is still under debate ${ }^{37,38}$. In case of positive margins, there is a consensus that the risk of the tumor recurring locally is high. However, there is less clear understanding related to negative margins definition, since there is fundamentally no consensus as to what an 'adequate' negative margin is. Thus, in practice some authors suggest that an absence of the tumor at the inked margin be in fact an adequate negative margin $^{39}$. In despite of that, comparing to negative margins, women with positive margins presented poorer 2- and 5-year breast cancer survival in Rio Branco, Acre. Early and latestage breast cancer patients with positive margins showed statistically poorer 2 -year survival, compared to the negative ones. Also, death risk was higher for women with positive margins, compared to free margins, regardless of age and stage. Such findings could be explained by the fact that residual disease at the surgical margins could increase the risk of local recurrence and possibly death through the years ${ }^{40}$.

Studies have shown higher risk of local recurrence even among women with breast cancer conserving surgery combined with radiotherapy $^{24}$. However, it is also true that some breast conserving therapy cases are treated without subsequent or post-operative radiotherapy ${ }^{38}$. In addition, not all residual carcinoma cells are necessarily radiation sensitive, and currently there are no established methods for determining the radiation responsiveness of individual patients ${ }^{38}$. Therefore, as expected, surgical margin status of the resected specimens is considered one of the most important factors related to the risk of local recurrence in individual patients.

Corroborating with other studies ${ }^{11,19}$, tumor size was one of the strongest predictor of survival in the present study. Although no statistical significance was observed when stratified by stage (Table-2), tumor size $\geq 2.5 \mathrm{~cm}$ increased the risk 
of death over 3-fold, as compared to tumor size $<2.5 \mathrm{~cm}$, even adjusted by age and stage. Many studies have suggested that lymph node status is independently associated with survival ${ }^{11,19}$. However, our finding suggest that even though early-stage patients presenting up to 2 lymph node involvement had better 2 -year breast cancer survival than those presenting over 2 lymph nodes involvement, no statistical significance was observed after being adjusted by age and stage.

Considering the small number of patients in this study and the high percentage of HER-2 missing values $(24.8 \%)$, we could not evaluate the combined effect of triple negative receptor tumors (ER-, PR- and HER2-), neither explore possible interaction between this hormone receptor subtype and prognostic factors such as stage, tumor size, age, nodes and treatments. Also, we could not evaluate breast cancer-specific 5-year survival rates stratified by possible confounding, neither estimate crude nor adjusted 5-year HR, because UNACON/Acre is a young cancer center allowing only a short follow-up time of recent cohorts. On the other hand, to our knowledge, this is the first breast-cancer survival study ever performed in the western Amazon area estimating prognostic value of clinical, epidemiological and molecular char- acteristics. Additionally, since this study is based on all hospitals and laboratories in the city of Rio Branco and only 6 patients were treated out of the city, the cases may be representative of all breast cancer patients in Rio Branco, Acre.

\section{Conclusion}

Hospital breast cancer specific-survival in Rio Branco, Acre, is similar to those found in developed countries $(\geq 80 \%)$, and even higher than those found in developing countries such as India (77\%) and Porto Rico (71,2\%). Also, 5-year breast cancer-specific survival in Rio Branco was similar to those found in south and southeastern area in Brazil. Almost two-third of breast cancers observed in the present study (58.4\%) was in early stage (0-II) at diagnosis. Late stage at diagnosis increased the risk of death in 2-years over 3-fold as compared to early stage, even adjusted by age. On the other hand, tumor size $\geq 2.5 \mathrm{~cm}$, positive margins, negative progesterone receptor and any other treatment but surgery combined with radiotherapy affected the 5-year breast cancer-specific survival, and increased the risk of death in two years.

\section{Collaborations}

RHP Fujimoto coordinated the study, participated in the collecting data, and statistical analysis; RJ Koifman participated in study design, data interpretation and final approval of the manuscript; IF Silva participated in study design, statistical analysis and interpretation of data and preparation of the manuscript.

\section{Acknowledgments}

The authors thank Dr. Sergio Koifman (in memoriam), by contributing to the vision on the assessment of breast cancer reality in Rio Branco, Acre, and open this path through impact studies on mortality, incidence and screening. 


\section{References}

1. Ferlay J, Shin HR, Bray F, Forman D, Mathers C, Parkin DM. GLOBOCAN 2012, Cancer Incidence and Mortality Worldwide. IARC Cancer Base 2012; No. 10. [cited 2014 Sep 9]. Available from: http://globocan.iarc.fr. Accessed 2014 Sep 09.

2. Hery C, Ferlay J, Boniol M, Autier P. Quantification of changes in breast cancer incidence and mortality since 1990 in 35 countries with Caucasian-majority populations. Ann Oncol 2008; 19(6):1187-1194.

3. Autier P, Hery C, Haukka J, Boniol M, Byrnes G. Advanced breast cancer and breast cancer mortality in randomized controlled trials on mammography screening. JCO 2009; 27(35):5919-5923.

4. Macià $\mathrm{F}$, Porta $\mathrm{M}$, Murta-Nascimento $\mathrm{C}$, Servitja $\mathrm{S}$, Guxens M, Burón A, Tusquets I, Albanell J, Castells $\mathrm{X}$. Factors affecting 5- and 10-year survival of women with breast cancer: an analysis based on a public general hospital in Barcelona. Cancer Epidemiol 2012; 36(6):554-559.

5. Peto R. The worldwide overview: new results for systemic adjuvant therapies. Breast Cancer Res 2007; 106:S5.

6. Ganesh B, Talole SD, Dikshit R, Badwe RA, Dinshaw KA. Estimation of survival rates of breast cancer patients - a hospital-based study from Mumbai. Asian Pac J Cancer Prev 2008; 9(1):53-57.

7. Ortiz AP, Frías O, Pérez J, Cabanillas F, Martínez L, Sánchez C, Capó-Ramos DE, González-Keelan C, Mora E, Suárez E. Breast cáncer molecular subtypes and survival in a hospital-based sample in Puerto Rico. Cancer Med 2013; 2(3):343-350.

8. Giedion U, Villar M, Ávila A, Fundation Mapfre. Los sistemas de Salud en Latinoamérica y el papel del Seguro Privado. Madrid: Instituto de Ciencias Del Seguro; 2010.

9. Mendonça GAS, Silva AM, Caula WM. Tumor characteristics and five-year survival in breast cancer patients at the National Cancer Institute, Rio de Janeiro, Brazil. Cad Saude Publica 2004; 20(5):1232-1239.

10. Moraes AB, Zanini RR, Turchiello MS, Riboldi J, Medeiros RL. Survival study of breast cancer patients treated at the hospital of the Federal University in Santa Maria, Rio Grande do Sul, Brazil. Cad Saude Publica 2006; 22(10):2219-2228.

11. Schneider IJC, D’Orsi E. Five-year survival and prognostic factors in women with breast cancer in Santa Catarina State, Brazil. Cad Saude Publica 2009; 25(6):1285-1296.

12. Martins DM, Vidal FC, Souza RD, Brusaca SA, Brito LM. Determination of CYP2D6 ${ }^{\star} 3,{ }^{*} 4$, and ${ }^{*} 10$ frequency in women with breast cancer in São Luís, Brazil, and its association with prognostic factors and disease-free survival. Braz J Med Biol Res 2014; 47(11):1008-1015.

13. Nakashima JP, Koifman RJ, Koifman S. Incidência de câncer na Amazônia ocidental: estimativa de base populacional em Rio Branco, Acre, Brasil, 2007-2009. Cad Saude Publica 2012; 28(11):2125-2132.
14. Nakashima JP, Koifman S, Koifman RJ. Cancer mortality trends in Rio Branco, Acre State, Brazil, 1980-2006. Cad Saude Publica 2011; 27(6):1165-1174.

15. Percy MJ, Myrie KA, Neeley CK, Azim JN, Ethier SP, Petty EM. Expression and mutational analyses of the human MAD2L1 gene in breast cancer cells. Genes Chromosomes Cancer 2000; 29(4):356-362.

16. World Health Organization (WHO). Meeting of Heads of WHO Collaborating Centers for the Classification of Diseases. Copenhagen: WHO; 1997.

17. Brazilian Society of Mastology (SBM). Tracking of Breast Cancer in Brazilian Women. Recommendations from a Consensus Meeting 10st, 2008, São Paulo. [cited 2014 Jun 06]. Available from: http://www.sbmastologia.com.br/download/reuniao_de_consenso_2008.pdf

18. BalabramD,Turra CM, Gobbi H. Survival of patients with operable breast cancer (Stages I-III) at a Brazilian public hospital - a closer look into cause-specific mortality. BMC Cancer 2013; 13:434.

19. Hosmer DW, Lemeshow S, May S. Applied survival analysis: regression modelling of time-to-event data. $2^{\text {nd }}$ ed. Hoboken: John Wiley \& Sons; 2008.

20. Carneseca EC, Mauad EC, Araujo MAA, Dalbó RM, Longatto Filho A, Vazquez VL. The Hospital de Cancer de Barretos Registry: an analysis of cancer survival at a single institution in Brazil over a 10-year period. BMC Research Notes 2013; 6:141.

21. Sant M, Allemani C, Berrino F, Coleman MP, Aereleid T, Chaplain G, Coebergh JW, Colonna M, Crosignani P, Danzon A, Federico M, Gafa L, Grosclaude P, Hédelin G, Macè-Lesech J, Garcia CM, Møller H, Paci E, Raverdy N, Tretarre B, Williams EM; European Concerted Action on Survival and Care of Cancer Patients (EUROCARE) Working Group. Breast carcinoma survival in Europe and the United States. Cancer 2004; 100(4):715-22.

22. Höfelmann DA, Anjos JC, Ayala AL. Survival for ten years and prognostic factors for women with breast cancer in Joinville in the State of Santa Catarina, Brazil. Cien Saude Colet 2014; 19(6):1813-1824.

23. Poum A, Kamsa-Ard S, Promthet S. Survival rates of breast cancer: a hospital-based study from northeast of Thailand. APJC 2012; 13(3):791-794.

24. Garicochea B, Morelle A, Andrighetti AE, Cancella A, Bós A, Werutsky. Ageas a prognostic factorin breastcancer early. Rev Saude Publica 2009; 43(2):311-317.

25. Hayes DF. Clinical practice. Follow-up of patients with early breast cancer. New Engl J Med 2007; 356(24):25052513.

26. Sundquist M, Thorstenson S, Brudin L, Wingren S, Nordenskjöld B. Incidence and prognosis in early onset breast cancer. Breast 2002; 11(1):30-35.

27. Soerjomataram I, Louwman MW, Ribot JG, Roukema JA, Coebergh JW. An overview of prognostic factors for long-term survivors of breast cancer. Breast Cancer Res Treat 2008; 107(3):309-330. 
28. Chitapanarux I, Trakultivakorn H, Srisukho S, Somwangprasert A, Watcharachan K, Srikawin J, Chaiwun B, Traisathit P. Real-world outcomes of different treatments in the management of patients with HER-2 positive breast cancer: a retrospective study. J Med Assoc Thai 2013; 96(6):709-715.

29. Jana D, Mandal S, Mukhopadhyay M, Mitra D, Mukhopadhyay SK, Sarkar DK. Prognostic significance of HER-2/neu and survival of breast cancer patients attending a specialized breast clinic in Kolkata, Eastern India. Asian Pac J Cancer Prev 2012; 13(8):3851-3855.

30. Kroman N, Jensen M, Wohlfahrt J, Mouridsen HT, Andersen PK, Melbye M. Factors influencing the effect of age on prognosis in breast cancer: population based study. BMJ 2000; 320(7233):474-478.

31. Liedtke C, Kiesel L. Breast cancer molecular subtypes--modern therapeutic concepts for targeted therapy of a heterogeneous entity. Maturitas 2012; 73(4):288-294.

32. Dowsett M, Allred C, Knox J, Quinn E, Salter J, Wale C, Cuzick J, Houghton J, Williams N, Mallon E, Bishop H, Ellis I, Larsimont D, Sasano H, Carder P, Cussac AL, Knox F, Speirs V, Forbes J, Buzdar A. Relationship between quantitative estrogen and progesterone receptor expression and human epidermal growth factor receptor 2 (HER-2) status with recurrence in the Arimidex, Tamoxifen, Alone or in Combination trial. J Clin Oncol 2008; 26(7):1059-1065.

33. Salmen J, Neugebauer J, Fasching PA, Haeberle L, Huober J, Wöckel A, Rauh C, Schuetz F, Weissenbacher T, Kost B, Stickeler E, Klar M, Orlowska-Volk M, Windfuhr-Blum M, Heil J, Rom J, Sohn C, Fehm T, Mohrmann S, Loehberg CR, Hein A, Schulz-Wendtland R, Hartkopf AD, Brucker SY, Wallwiener D, Friese K, Hartmann A, Beckmann MW, Janni W, Rack B. Pooled analysis of the prognostic relevance of progesterone receptor status in five German cohort studies. Breast Cancer Res Treat 2014; 148(1):143-151.

34. Shyamala G, Chou YC, Louie SG, Guzman RC, Smith GH, Nandi S. Cellular expression of estrogen and progesterone receptors in mammary glands: regulation by hormones, development and aging. J Steroid Biochem Mol Biol 2002; 80(2):137-148.

35. Medina D, Kittrell FS, Shepard A, Contreras A, Rosen JM, Lydon J. Hormone dependence in premalignant mammary progression. Cancer Res 2003; 63(5):10671072.

36. Hoefnagel LD, Moelans CB, Meijer SL, van Slooten HJ, Wesseling P, Wesseling J, Wesseling J, Westenend PJ, Bart J, Seldenrijk CA, Nagtegaal ID, Oudejans J, van der Valk P, van Gils CH, van der Wall E, van Diest PJ. Prognostic value of estrogen receptor alpha and progesterone receptor conversion in distant breast cancer metastases. Cancer 2012; 118(20):4929-4935.
37. Behm EC, Beckmann KR, Dahlstrom JE, Zhang Y, Cho C, Stuart-Harris R, Craft P, Rezo A, Buckingham JM. Surgical margins and risk of locoregional recurrence in invasive breast cancer: an analysis of 10-year data from the breast cancer treatment quality assurance project. Breast 2013; 22(5):839-844.

38. Ishida $T$, Takeda M, Suzuki A, Amari M, Moriya $T$, Ohuchi N. Significance of irradiation in breast-conserving treatment: comparison of local recurrence rates in irradiated and nonirradiated groups. Int J Clin Oncol 2008; 13(1):12-17.

39. Kaufmann M, von Minckwitz G, Mamounas EP, Cameron D, Carey LA, Cristofanilli M, Denkert C, Eiermann W, Gnant M, Harris JR, Karn T, Liedtke C, Mauri D, Rouzier R, Ruckhaeberle E, Semiglazov V, Symmans WF, Tutt A, Pusztai L. Recommendations from an international consensus conference on the current status and future of neoadjuvant systemic therapy in primary breast cancer. Ann Surg Oncol 2012; 19(5):1508-1516.

40. Al-Himdani S, Timbrell S, Tan KT, Morris J, Bundred NJ. Prediction of margin involvement and local recurrence after skin sparing and simple mastectomy. Eur J Surg Oncol 2016; 42(7):935-941.
Artigo apresentado em 22/07/2016

Aprovado em 06/02/2017

Versão final apresentada em 08/02/2017 
